\title{
Particle-in-cell Simulations of Raman Forward Scattering Instability in Low-density Plasmas: A Computational Study
}

\author{
Alire za Heidari, Se ye dali Vedad Fate meh Ghorbani, Mohammadali Ghorbani* \\ Institute for Advanced Studies, Tehran, 14456-63543, Iran
}

\begin{abstract}
Electromagnetic-wave propagation through equilibrium plasmas can lead to medium instabilities whose formation and growth conditions are of importance to study. Raman forward scattering (RFS) instability in low-density plasmas is investigated through particle-in-cell (PIC) simu lation, which is employed due to plasmas' high complexity, costly experiment process, and nonlinear behavior in physical terms. A 2D electromagnetic PIC code is used to model RFS instability. Do ing so, a plasma medium containing ions and electrons is PIC simulated and ions are assumed to form a constant background because of high inertia. The results indicate that as a plane electromagnetic wave enters the plasma med iu m, large-a mp litude longitudinal waves, whose growth rate is well consistent with the theoretical results, begin to grow, suggesting the appearance of RFS instability.
\end{abstract}

Keywords Low-Density Plas ma, PIC Simulation, RF S Instability

\section{Introduction}

Various parameters such as instabilities are significant to describe equilibrium plasmas. A system is in instable equilibrium whenever there is a growing perturbation affecting the system's total configuration or macroscopic quantities. In a simple classification, the instabilities are divided into two main categories of position-space instabilities (macroscopic) and velocity-space instabilities (microscopic), which include electrostatic and electromagnetic instabilities. A plasma current creates a magnetic field shrinking plasma and increasing current density, which causes electromagnetic instability. In this instance, $\vec{\nabla} \times \vec{E} \neq 0$ and the set of Maxwell equations must be comp letely solved[1].

Here, we deploy computer simulation to investigate plasma instabilities. This method is classified as a computational science in which the start point is to scientifically study the mathematical model of the phenomenon. The equations of the mathe matical model must become algebraically discrete in order to be understandable in terms of numerical solution. When the discrete equations are expressed as a series of computer commands, they describe the simulation model as a computer simulation program. A mass of data is produced even by the simplest

* Corresponding author:

mohammadalighorbani1983@yahoo.com (Mohammadali Ghorbani)

Published online at http://journal.sapub.org/ijtmp

Copyright (C) 2012 Scientific \& Academic Publishing. All Rights Reserved simulation calculations. Hence, most research efforts in this field have been focused to obtain appropriate simulation methods for physical systems that can be investigated using available co mputer resources. The main difference between a simulated plasma and a real plasma is in the number of charges, fields, and spatial/temporal criteria. A real plas ma is composed of a multitude of positive electrons and ions. In a simulation process, a charged particle is essentially a homogeneous ensemble of numerous charged particles existing in a real plas ma. Therefore, its charge and mass is several times larger than real particles'. However, the simulated particles' charge/mass ratio is the same as that of real particles; consequently, we can substitute a lower number of charges in simulation and simulate physical phenomena by a limited number of particles. One of the main advantages of simulation is the low volume of arrays and calculations. The application of the word "particle" in computer memory represents the concept of charged particles occupying the real space.

Note that, many theoretical investigations regarding Raman instability have been well carried out and this paper aims to reconcile the PIC simulation results with the theoretical results.

\section{RFS Instability and the Used Equations}

In Raman instability, an input large-amplitude light wave is transformed into a scattered light wave and a plasma electron wave. The phase velocity in direct forward 
scattering is close to the speed of light and there are a tiny number of particles in the plasma background, absorbing energy required to be captured by these waves and accelerated consequently. If the input wave is propagated through a low-density plasma in a relatively long distance, a large-amplitude plas ma electron wave will be created that can produce high-energy electrons [2].

An input wave $\left(\omega_{0}, k_{0}\right)$ in the form of $\vec{E}=\vec{E}_{0} \cos \left(k_{0}-\omega_{0} t\right)$ is transformed into a scattered wave $\left(\omega_{s}, k_{s}\right)$ and a plasma electron wave $\left(\omega_{e k}, k_{p}\right)$ that follow the equations below[3]:

$$
\omega_{s}^{2}=\omega_{p}^{2}+k_{s}^{2} c^{2}, \quad \omega_{e k}^{2}=\omega_{p}^{2}+3 k_{p}^{2} v_{e}^{2}
$$

where $v_{e}=\sqrt{k_{B} T / m_{e}}$ is the thermal velocity of the plasma electrons, $k_{B}$ is the Boltzmann constant, and $m_{e}$ denotes the electron mass. The frequency and wavenumber matching (phase matching) conditions are defined as:

$$
\omega_{0}=\omega_{s}+\omega_{e k}, \quad \vec{k}_{0}=\vec{k}_{s}+\vec{k}_{p}
$$

where $\omega_{0}\left(\omega_{s}\right)$ and $\vec{k}_{0}\left(\vec{k}_{s}\right)$ respectively represent the frequency and wavenumber of the incident (scattered) light wave, and $\omega_{e k}\left(\vec{k}_{p}\right)$ denotes the frequency (wavenumber) of the plasma electron wave.

The maximu m growth rate of stimu lated Raman backward and forward scattering (SRS) in homogeneous plasma is obtained from[4]:

$$
\gamma=\frac{k_{p} v_{o s}}{4}\left[\frac{\omega_{p e}^{2}}{\omega_{e k}\left(\omega_{0}-\omega_{e k}\right)}\right]^{\frac{1}{2}}
$$

A wave must penetrate into the plasma in order to create Raman instability. Since the minimum frequency of a light wave in plasma is $\omega_{p e}$ (plasma electron frequency), it is obvious that $\omega_{0} \geq 2 \omega_{p e}$, based on the matching conditions. It means $n \leq n_{c r} / 4$, where $n$ denotes plasma density and $n_{c r}$ represents critical plasma density[5]. Considering the plasma dispersion relation and frequency, we obtain $n_{c r}=m \omega_{0}^{2} / 4 \pi e^{2}$. It is called low-density plasma if $n \leq n_{c r} / 4$.

\section{PIC Simulation Method}

PIC simulation is employed to trace the trajectory of charged particles, which are calculated on constant-lattice points, in a self-consistent electromagnetic field. The simulation outline is based on the concept that fields are self-consistent. If the distribution of particles at each time step and the values of the fields at the previous time step are known, the values of the field at the new time step can be determined using the equations governing the field (a mpere-faraday). Such a field affects the motion of particles and rearranges them. This effect can be determined using the Lorentz force solution. Accordingly, these electromagnetic fields and the distribution of particles a ffect each other. It is the self-consistency of electromagnetic fields. The general algorithm of the written 2D electromagnetic PIC code is defined as follows:

1) First, the program of the in itial conditions is written by determining the particles' position and velocity. Then, various parameters such as charge density and current density can be obtained in each time interval and on each lattice point through interpolation. By obtaining charge density and current density, the electromagnetic fields on the lattice points can be calculated by the field equations.

2) The position of the phase points in the phase space is determined through the Newton-Lorentz equations. However, to do such calculations, we need to have the values of the fields on the phase points, which are obtained by extrapolating the values of the fields on the lattice points[6]. The values obtained for the position and velocity of the phase points are used as the initial conditions of the next time step, and all the program steps will fo llow the same procedure. All the above stages are written in a $\mathrm{C}++$ code.

There are some criteria to ensure that the code is properly implemented in terms of the number of particles, temporal interval, and spatial interval. The number of particles should be much higher than that of lattice points, which ensures the fact that all the lattice squares constantly averagely contain several particles during the simulation process. The spatial interval should not exceed the Debye length $\left(\lambda_{D}\right)$ in order for the distribution of potential to be computable by charge separation. The temporal interval should be lesser than the inverse plasma electron frequency $\left(\omega_{p e}^{-1}\right)$ in order to produce plasma oscillations[7].

The first step to calculate a system of equations is to make them dimensionless to avoid the errors due to very large or small coefficients. The nondimensionalization of the 2D code with respect to the input wave's properties is defined as:

$$
\begin{aligned}
& t \omega_{0} \rightarrow t, \quad k_{0} x \rightarrow x, \quad \frac{v}{c} \rightarrow v, \\
& \frac{q}{m c \omega_{0}} E(B) \rightarrow E(B), \quad \frac{4 \pi q}{m c \omega_{0}^{2}} J \rightarrow J
\end{aligned}
$$

where $\omega_{0}$ and $k_{0}$ respectively denote the frequency and wavenumber of the input electromagnetic plane wave, $m$ and $q$ are the mass and electric charge of particles respectively, $E$ is the electric field, $B$ is the magnetic field, $c$ is the speed of light in vacuum, $J$ is the current density, $v$ is the velocity of particles, $x$ is the position of particles, and $t$ represents time.

The interaction between an electromagnetic plane wave and a plasma lobe can be simulated through the Maxwell equations, which are defined in the Gaussian systemas: 


$$
\begin{aligned}
& \vec{\nabla} \times \vec{B}=\frac{1}{c} \frac{\partial \vec{E}}{\partial t}+\frac{4 \pi \vec{J}}{c}, \quad \vec{\nabla} \times \vec{E}=-\frac{1}{c} \frac{\partial \vec{B}}{\partial t}, \\
& \vec{\nabla} \cdot \vec{E}=4 \pi \rho, \quad \vec{\nabla} \cdot \vec{B}=0
\end{aligned}
$$

Solving these equations in three dimensions leads to a quite complex ensemble of arrays and calculations. Hence, we try to simplify the calculations through reducing the problem's dimensions. Here, we study the evolution of an electromagnetic plane wave with linear polarization propagated along the $\mathrm{x}$-axis in an interaction with plasma. Accordingly, the vector potential components become $\vec{A}=\left(0, A_{y}, 0\right) ;$ given that $\vec{E}=-\vec{\nabla} \varphi-\partial \vec{A} / \partial t$ and $\vec{B}=\vec{\nabla} \times \vec{A}$, the electromagnetic-field components are defined as $E_{x}, E_{y}$, and $B_{z}$.

While a plane wave with such properties is passing, if the electrons do not have any initial velocity along the $z$-axis, the motion of the particles will remain in the xy-plane and there will not be any evolution along the z-axis (Appendix A).

\section{Initial Conditions and the Simulation Model}

The initial conditions and dimensionless data we used in the 2D electromagnetic code are as follows:

The length of the simulation box along the $\mathrm{x}$-axis and $\mathrm{y}$-axis is chosen 50 , the spatial distance between the lattice points along the $\mathrm{x}$-axis and $\mathrm{y}$-axis is 0.1 , there is one particle in each cell, the total number of particles is $2 \times 10^{5}$, the number of lattice points along the $\mathrm{x}$-axis and $\mathrm{y}$-axis is 501 , the amplitude of the incident electric wave is 0.05 , the wavelength of the incident wave is 6 , the incident-wave wavenumber is $100 / 3$, the frequency of the dimensional incident wave is $10^{12}\left(\mathrm{sec}^{-1}\right)$, the initial density of the dimensional plasma is $1.23 \times 10^{10}\left(\mathrm{~cm}^{-1}\right)$, and the dimensional-plas ma electron frequency is $6.1 \times 10^{9}\left(\mathrm{sec}^{-1}\right)$.

Considering the periodic conditions along the y-axis, the particles are assumed to be immobile electrons and placed within $5<x<45$. The spatial distribution of electrons is homogeneous. The plasma medium containing electrons and ions is simulated by the PIC method, and ions are as sumed to form a constant background, owing to high inertia. However, the dynamics of the electrons is obtained through Newton-Lorentz equations. After calculation, we obtain $n=1.23 \times 10^{10}$ and $n_{c r}=3.1 \times 10^{13}$. Given $n \leq n_{c r} / 4$, instability is expected to appear in the code results. The time steps are determined through $\mathrm{C}++$. The program operation period is calculated by the number of iteration.

Given an input electromagnetic plane wave with $\vec{E}=E_{0} \sin (\omega t-k x) \hat{j}$ entering the right-handed boundary $(x=0)$ of the simulation box, and applying $\vec{B}=E_{0} \sin (\omega t-k x) \hat{k}$ to the same boundary according to the Maxwell equations, we investigate longitudinal-wave growth or RFS instability. Note that the theoretical investigations in this field have been previously performed and this paper aims to compare those results with the results obtained from the PIC simulation.

\section{Results and Discussion}

The simu lation box should be initially empty of plas ma to compare the transmission of the plane wave through plasma and vacuum. It can be shown that the propagation of an electromagnetic plane wave through the vacuum is in compliance with the theoretical results and there is not any longitudinal electric wave. These numerical results suggest this code's proper performance[8].

Now, regarding RFS instability, considering the initial conditions and the simulation model, the electromagnetic plane wave first enters the vacuum boundary, and then enters the low-density plasma slab. This wave returns the vacuum region after passing this slab.

Here we consider a thin plasma slab and investigate the propagation of electromagnetic waves through it. Given an input plane wave $\left(\vec{E}=E_{0} \sin (\omega t-k x) \hat{j}\right)$ applying to the right-handed boundary ( $x=0)$ of the simulation box, we study RFS instability or longitudinal-wave growth through plasma.

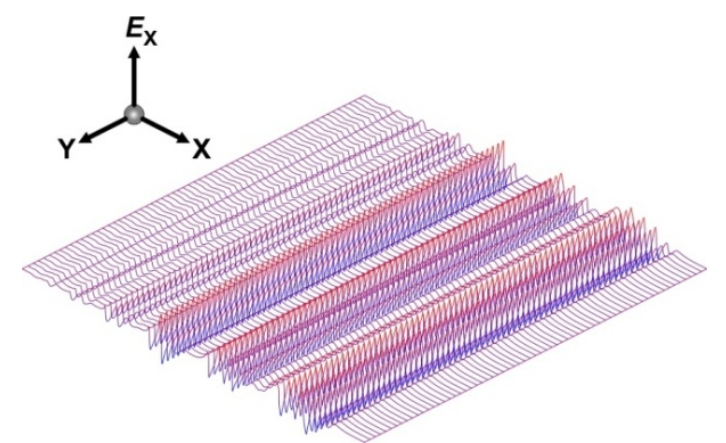

Figure 1. Longitudinal electric waves due to input-wave propagation through plasma

Figure 1 shows how the longitudinal electric field grows due to the propagation of the input wave through plasma (the number of iteration is 5000). As evident, opposed to the vacuum state, the longitudinal electric field starts to grow and reaches the maximum of $5 \times 10^{-4}$, which is considerable in comparison to the input-wave amplitude. The maximum longitudinal-wave amplitude is grown approximately one hundredth of the input-wave amp litude.

Given that $n \leq n_{c r} / 4$ in this code, the plasma is expected to be transparent and the input wave easily passes through it. The correctness of this case has been already proved.

Note that the growth of the longitudinal-wave amp litude is limited by turbulence resulting from holes in plasma. This is sue implies the plasma nonlinear effects [9-11]. In this state, the particles captured by the wave continuously exchange 
energy with the wave. As can be seen in Figure 1, the longitudinal wave deteriorates as it progresses.

However, since the density of the plasma particles is still much lesser than the critical density, the plasma is transparent and the propagation of the input wave through plasma is the same as what Figure 2 shows.

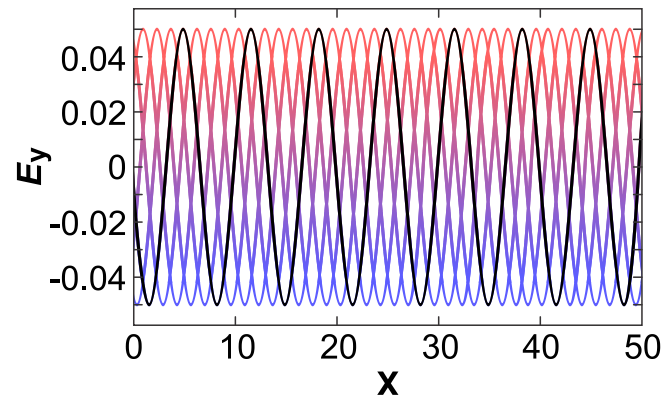

Figure 2. Transverse electric waves due to input-wave propagation through plasma

The growth rate of instability is the most important parameter that should be measured. Figure 3 indicates the logarith mic diagram of the longitudinal electric field versus time. As can be observed, although it fluctuates, the field amp litude is substantial and shows a gentle trend for $t>25$. The slope of the diagram at this reg ion can be considered as the field growth rate. Linear least-square fitting is the best technique to calculate the slope of this diagram. The slope of this diagram or the instability growth rate is obtained $1.0127 \times 10^{-3}$, showing a $12.52 \%$ deviation from the theoretical result $\left(0.9 \times 10^{-3}\right)$ obtained fro $\mathrm{m} \mathrm{Eq.} \mathrm{(3).}$

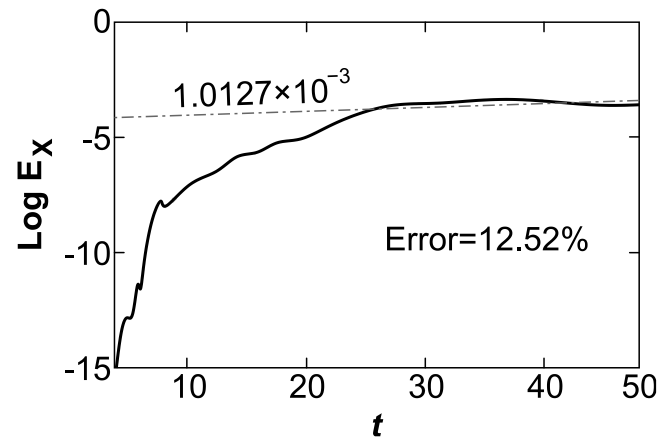

Figure 3. The logarithmic diagram of Longitudinal electric field vs. time

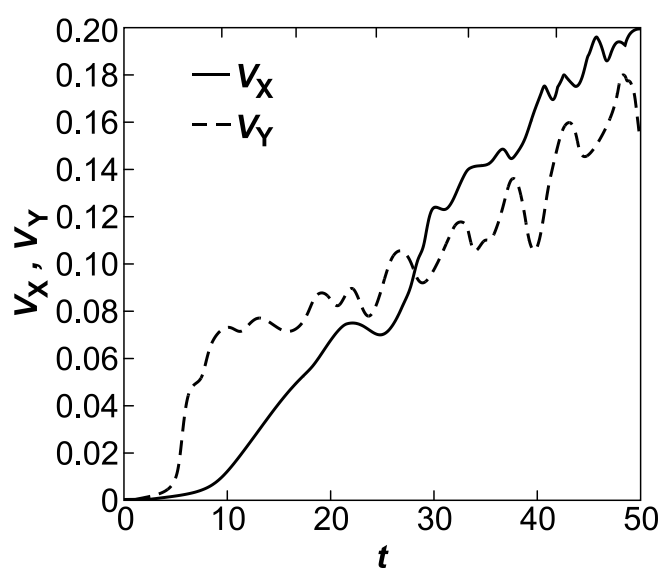

Figure 4. Velocity along $x-a x i s$ and $y$-axis vs. time
Regarding the phase space, Figure 4 shows the maximum velocity along the $\mathrm{x}$-axis and $\mathrm{y}$-axis in each temporal interval for a 50-length simulation box and 5000 iteration steps. As evident, the velocity rises along both $\mathrm{x}$-axis and $\mathrm{y}$-axis, and declines for the next temporal intervals.

\section{Conclusions}

In this paper, RFS instability was simulated by a $2 \mathrm{D}$ electro magnetic PIC code written by $\mathrm{C}++$. First, because we were sure about this code's proper performance, the propagation of electromagnetic waves through vacuum and low-density plasma was investigated. We found that if the medium is a low-density plasma, there will not be a difference in the propagation of electromagnetic waves through plasma and vacuum. It means that a low-density plasma acts as a transparent medium against electromagnetic waves. Also, it was observed that large-amplitude longitudinal waves are produced and grown when a plane wave passes through a low-density plasma. This case indicates instability in the propagation of electromagnetic waves through low-density plasmas. The slight difference between the calculated growth rate and the theoretical growth rate suggests this code's proper performance.

\section{Appendix}

\section{A. Simulation of field equations}

The Maxwell equations govern fields and electromagnetic waves, whereby we can study the evolution of electromagnetic fields in time and position. Given the spatial-temporal dependence of electromagnetic fields (Faraday's law and Ampère-Maxwell law), the discretization of these dimensionless equations $(\partial \vec{B} / \partial t=-\vec{\nabla} \times \vec{E}$ and $\partial \vec{E} / \partial t=\vec{\nabla} \times \vec{B}-\vec{J})$ is defined as the following procedure.

Considering the above equations (the temporal derivation of $\vec{E}$ and $\vec{B}$ at the left-handed side and their existence in the right-handed side), the temporal derivation has been performed using the leap-frog scheme in integer temporal intervals and temporal and spatial semi-integer intervals [11].

It is the time centering method and the accuracy of the temporal derivatives is of the second order. In the 2D space, fields are divided into transverse electric (TE) and transverse magnetic (TM) fields. All the spatial variables, including $\vec{K}$, are on the xy-plane. Assuming $\vec{k} \cdot \vec{B}=0$ for the TM fields, we have $E_{x}, E_{y}$, and $B_{z}$. Assuming $\vec{k} \cdot \vec{E}=0$ for the TE fields, we have $B_{x}, B_{y}$, and $E_{z}$. The value of TE fields should not necessarily be calculated and saved since the TE fields remain zero in some applications[12].

Now, employing the Morse-Nielson discretization method, the temporal derivative of the Maxwell equations can be written as: 


$$
\left(\partial_{t} E_{x}\right)_{j+\frac{1}{2}, k}^{n+\frac{1}{2}}=\frac{\left(E_{x}\right)_{j+\frac{1}{2}, k}^{n+1}-\left(E_{x}\right)_{j+\frac{1}{2}, k}^{n}}{\Delta t}
$$

such that

$$
\left(E_{x}\right)_{j+\frac{1}{2}, k}^{n}=E_{x}\left(\left[j+\frac{1}{2}\right] \Delta x, k \Delta y, n \Delta t\right)
$$

where $\left(E_{x}\right)_{j+(1 / 2), k}^{n}$ denotes the x-component of the electric field at $n \Delta t, j+(1 / 2) \Delta x$, and $k \Delta y$. Consequently, the Maxwell equations of the TM fields are defined as follows[12]:

$$
\begin{gathered}
\left(\partial_{t} B_{z}\right)_{j+\frac{1}{2}, k+\frac{1}{2}}^{n}=-\left(\partial_{x} E_{y}-\partial_{y} E_{x}\right)_{j+\frac{1}{2}, k+\frac{1}{2}}^{n} \\
\left(\partial_{t} E_{x}\right)_{j+\frac{1}{2}, k}^{n+\frac{1}{2}}=\left(\partial_{y} B_{z}-J_{x}\right)_{j+\frac{1}{2}, k}^{n+\frac{1}{2}} \\
\left(\partial_{t} E_{y}\right)_{j, k+\frac{1}{2}}^{n+\frac{1}{2}}=\left(-\partial_{x} B_{z}-J_{y}\right)_{j, k+\frac{1}{2}}^{n+\frac{1}{2}}
\end{gathered}
$$

For example, the extension of (A.3) is obtained as:

$$
\begin{aligned}
& \frac{\left(B_{z}\right)_{j+\frac{1}{2}, k+\frac{1}{2}}^{n+\frac{1}{2}}-\left(B_{z}\right)_{j+\frac{1}{2}, k+\frac{1}{2}}^{n-\frac{1}{2}}}{\Delta t} \\
= & -\frac{\left(E_{y}\right)_{j+1, k+\frac{1}{2}}^{n}-\left(E_{y}\right)_{j, k+\frac{1}{2}}^{n}}{\Delta x}+\frac{\left(E_{x}\right)_{j+\frac{1}{2}, k+1}^{n}-\left(E_{x}\right)_{j+\frac{1}{2}, k}^{n}}{\Delta y}
\end{aligned}
$$

Assuming that $B_{z}^{n-(1 / 2)}$ and $E_{x, y}^{n}$ are known in (A.6), $B_{z}^{n+(1 / 2)}$ is obtained. Accordingly, we can determine the electric-field components at the next temporal interval $\left(E_{x}^{n+1}, E_{y}^{n+1}\right)$, and obtain the components of the electric and magnetic fields at all the time steps.

The plasma partic les do not have any initial velocity at the first step. Such that a laser beam radiates into a solid and makes it plasma. To calculate the magnetic field in the first temporal semi-interval, we use the equation below:

$$
\vec{B}^{n}=\vec{B}^{n-\frac{1}{2}}-\frac{c \Delta t}{2} \vec{\nabla} \times \vec{E}
$$

As the equations indicate, the electric and magnetic fields are calculated at different time steps. The magnetic fields are calculated in the temporal semi-integer intervals $\left(B_{z}^{n \pm(1 / 2)}\right)$. The electric fields are calculated at the ends of the temporal integer intervals $\left(E_{x}^{n}, E_{x}^{n+1}, E_{y}^{n}\right.$, and $\left.E_{y}^{n+1}\right)$. Since the fields should be necessarily the same in the motion of particles, we average $B_{z}^{n}$ as[12]:

$$
\left(B_{z}\right)_{j+\frac{1}{2}, k+\frac{1}{2}}^{n}=\frac{\left(B_{z}\right)_{j+\frac{1}{2}, k+\frac{1}{2}}^{n+\frac{1}{2}}+\left(B_{z}\right)_{j+\frac{1}{2}, k+\frac{1}{2}}^{n-\frac{1}{2}}}{2}
$$

Now, the values of current density and charge density should be obtained considering the Maxwell equations. To do so, a weight function is applied to the lattice points as [12]:

Assuming that the $i^{\text {th }}$ charged particle is placed on $x_{j}$ and $y_{k}$, using the particles' current density and charge density, a spatial lattice in which $x_{j}=j \Delta x$ and $y_{k}=k \Delta y$ is considered to obtain the fields. This lattice's size, which is obtained from $\Delta x$, should be small enough to avoid numerical errors. To calculate each particle's current and charge density, we consider a surface weight function. These weights are given by:

$$
\begin{gathered}
\rho_{j, k}=\rho_{c} \frac{(\Delta x-x)(\Delta y-y)}{\Delta x \Delta y}, \quad \rho_{j+1, k}=\rho_{c} \frac{x(\Delta y-y)}{\Delta x \Delta y}, \\
\rho_{j+1, k+1}=\rho_{c} \frac{x y}{\Delta x \Delta y}, \quad \rho_{j, k+1}=\rho_{c} \frac{(\Delta x-x) y}{\Delta x \Delta y}
\end{gathered}
$$

where $\rho_{c}=q /$ Area is one cell's charge density. The Boris method to calculate the current density is defined as:

$$
J_{j}^{n+\frac{1}{2}}=\sum_{i} v_{i}^{n+\frac{1}{2}} \frac{S\left(X_{j}-x_{i}^{n}\right)+S\left(X_{j}-x_{i}^{n+1}\right)}{2}
$$

whereby the average we ight function of the surface $S$ at the positions $x^{n}$ and $x^{n+1}$ is multiplied by $v^{n+(1 / 2)}$ and the current density $J^{n+(1 / 2)}$ is obtained, consequently. The four nearest points surrounding each particle are used to calculate that particle's current density[12].

\section{B. Simulation of single-particle 2D motion}

The following Newton-Lorentz equation is employed to study the motion of particles.

$$
m \frac{d \vec{v}}{d t}=q(\vec{E}+\vec{v} \times \vec{B})
$$

which can be solved by three simultaneous scalar equations. However, the Boris method is simpler and of the second order in which the Newton-Lorentz equation is discretized as:

$$
\frac{\vec{v}^{n+\frac{1}{2}}-\vec{v}^{n-\frac{1}{2}}}{\Delta t}=\frac{q}{m}\left[\vec{E}^{n}+\frac{\vec{v}^{n+\frac{1}{2}}+\vec{v}^{n-\frac{1}{2}}}{2} \times \vec{B}^{n}\right]
$$

where $m$ denotes the particle rest mass (electron or ion). In this method, because the electric and magnetic fields are investigated separately, there are two virtual velocities $v^{+}$ and $v^{-}$defined as: 


$$
\vec{v}^{n-\frac{1}{2}}=\vec{v}^{-}-\frac{q \vec{E}^{n}}{m} \frac{\Delta t}{2}, \quad \vec{v}^{n+\frac{1}{2}}=\vec{v}^{+}+\frac{q \vec{E}^{n}}{m} \frac{\Delta t}{2}
$$

Inserting (B.3) into (B.2), we obtain:

$$
\frac{\vec{v}^{+}-\vec{v}^{-}}{\Delta t}=\frac{q}{2 m}\left(\vec{v}^{+}+\vec{v}^{-}\right) \times \vec{B}^{n}
$$

which suggests that the vector $\left(\vec{v}^{+}+\vec{v}^{-}\right)$is perpendicular to the vector $\left(\vec{v}^{+}-\vec{v}^{-}\right)$, and $\left|\vec{v}^{-}\right|=\left|\vec{v}^{+}\right|$. The transformation $\vec{v}^{-} \rightarrow \vec{v}^{+}$is a $\theta$-angle rotation, which is defined as:

$$
\left|\tan \frac{\theta}{2}\right|=\left|\frac{v_{\perp}^{+}-v_{\perp}^{-}}{v_{\perp}^{+}+v_{\perp}^{-}}\right|=\frac{q B}{m} \frac{\Delta t}{2}=\omega_{c} \frac{\Delta t}{2}
$$

where $\omega_{c}=q B / m$ represents the cyclotron frequency [12]. To perform this rotation, we define two auxiliary vectors $t$ and $s$ as follows:

$$
\vec{t}=-\frac{q \vec{B}}{m} \frac{\Delta t}{2}, \quad \vec{s}=\frac{2 \vec{t}}{1+|t|^{2}}
$$

Emp loying (B.6), we have:

$$
\vec{v}^{\prime}=\vec{v}^{-}+\vec{v}^{-} \times \vec{t}, \quad \vec{v}^{+}=\vec{v}^{-}+\vec{v}^{\prime} \times \vec{s}
$$

where $\vec{v}^{\prime}$ is a vector perpendicular to the vectors $\left(\vec{v}^{+}-\vec{v}^{-}\right)$and $\vec{B}$. Therefore, the Boris method is composed of three steps[12]:

1) The electric field affects $\vec{v}^{n-(1 / 2)}$ in the temporal semi-interval, and $\vec{v}^{-}$is obtained.

2) The magnetic field affects $\vec{v}^{-}$, and $\vec{v}^{+}$is obtained through rotation.

3) The electric field affects $\vec{v}^{+}$, and $\vec{v}^{n+(1 / 2)}$ is obtained.

Accordingly, velocity at the moment $n+(1 / 2)$ can be obtained by knowing the fields at $n$ and velocity at $n-(1 / 2)$.

\section{ACKNOWLEDGMENTS}

The work described in this paper was fully supported by grants from the Institute for Advanced Studies of Iran. The authors would like to express genuinely and sincerely thanks and appreciated and their gratitude to Institute for Advanced Studies of Iran.

\section{REFERENCES}

[1] N. A. Krall and A. W. Trivelpiece, "Principles of Plasma Physics". New York and London. McGRAW-HILL Book Company (1992).

[2] S. C. Wilks, W. L. Kruer, K. Estabrook, and A. E. Langdon, Phys. Fluids B 4 (9) (1992).

[3] C. Hugh Barr and F. Francis Chen, Phys. Fluids 30, 4 (1987) 1.

[4] W. L. Kruer, "The Physics of Laser plasma Interactions". California and New York, Addison-Wesley Publishing Company (1988) 1.

[5] S. C. Wilks, W. L. Kruer, K. Estabrook, and A. B. Longdon, Phys. Fluids B, 4, 9 (1992) 1.

[6] W. H. Press, S. A. Teukolsky, W. T. Vetterling, and B. P. Flannery, "Numerical Recipes", third edition (2007).

[7] M. Masek and K. Rohlena, Communication in Nonlinear Science and Numerical Simulation 13 (2008) 125.

[8] S. Brunner and E. J. Valeo, Phys. Rev. Lett., 93, 14 (2004) 3.

[9] R. W. Hockney and J. W. Eatwood. "Computing Simulation Using Particles". Institute of Physics Publishing Bristol and Philadelphia (1994).

[10] C. K. Birdsall and A. B. Longdon, "Plasma Physics Via computer Simulation". Institute of Phy sics Publishing Bristol and Philadelphia (1995).

[11] A. Heidari, A. Nabatchian, M. Zeinalkhani, M. Ghorbani, Applied Phy sics Research, 4, 2 (2012) 29. 